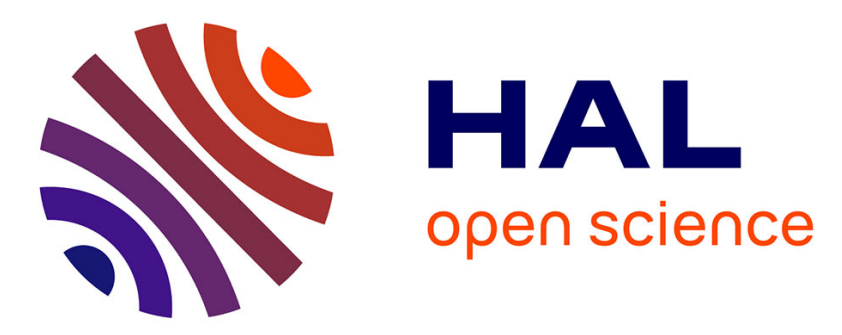

\title{
Bifurcation analysis of the control mechanism of pacemaker activity in a mathematical cardiac cell model
}

Karima Djabella, Mayer Landau, Michel Sorine

\section{To cite this version:}

Karima Djabella, Mayer Landau, Michel Sorine. Bifurcation analysis of the control mechanism of pacemaker activity in a mathematical cardiac cell model. 7th IFAC Symposium on Nonlinear Control Systems - NOLCOS'07, Aug 2007, Pretoria, South Africa. inria-00134529

\section{HAL Id: inria-00134529 \\ https://inria.hal.science/inria-00134529}

Submitted on 5 Mar 2007

HAL is a multi-disciplinary open access archive for the deposit and dissemination of scientific research documents, whether they are published or not. The documents may come from teaching and research institutions in France or abroad, or from public or private research centers.
L'archive ouverte pluridisciplinaire HAL, est destinée au dépôt et à la diffusion de documents scientifiques de niveau recherche, publiés ou non, émanant des établissements d'enseignement et de recherche français ou étrangers, des laboratoires publics ou privés. 


\title{
BIFURCATION ANALYSIS OF THE CONTROL MECHANISM OF PACEMAKER ACTIVITY IN A MATHEMATICAL CARDIAC CELL MODEL
}

\author{
Karima Djabella* Mayer Landau ** Michel Sorine* \\ * INRIA-Rocquencourt. BP 10578153 Le Chesnay Cedex, \\ France \\ ** CNRS, Laboratoire de Statistique et Génome, Université \\ d'Evry, France
}

\begin{abstract}
In this paper, a bifurcation analysis of a recently proposed reduced order cardiac cell model is performed to obtain insights into the mechanisms of control of the sinoatrial node pacemaker activity. The objective is to improve the understanding of cardiac arrhythmias caused by abnormal automaticity. The bifurcation structure of the model is studied as a function of the maximal conductances of six sarcolemmal currents: the sodium, calcium, background calcium and potassium currents, the sodium potassium pump and the sodium calcium exchanger. All these parameters, except for the sodium current, allow when varied the annihilation of the physiological rhythmic activity through Hopf bifurcation points. The background calcium current seems to be the current responsible for cycle length sensitivity in the physiological zone and then it appears as the main control input of the pacemaker activity.
\end{abstract}

Keywords: cardiac pacemaker cell, nonlinear dynamics, oscillation control, bifurcation analysis, computer simulation.

\section{INTRODUCTION}

The pacemaker activity of the sinoatrial (SA) node cells is an important subject in cardiac electrophysiology as the spontaneous activity controls the heart rate and is itself under the control of the autonomic nervous system (ANS) through different ionic channels.

The precise ionic currents that control membrane potential $(\mathrm{V})$ through gate mechanisms (g) during diastole and then the heart rate, are still a matter of debate (Kodama et al., 2002). (DiFrancesco, 1993) argues that the hyperpolarization activated current $\left(i_{f}\right)$ is the only current that can generate and control the slow depolarization of pacemaker cells. This current is normally carried by $\mathrm{Na}^{+}$and $\mathrm{K}^{+}$. (Guo et al., 1995) reported another current, called the sustained inward current $i_{s t}$, where the major charge carrier is believed to be $\mathrm{Na}^{+}$. Also a $\mathrm{Ca}^{2+}$ "window" current has been observed in rabbit sinoatrial node cells (Boyett et al., 2001). It is possible that any of these currents, or a combination of them, is responsible for membrane depolarization during diastole. In our simulations using a new reduced order model of the SA node cell (Djabella and Sorine, 2006), we concluded that all the currents directly or indirectly contribute to pacemaker depolarization, with the background calcium current $\left(I_{b, C a}\right)$ being the most dominant inward current during diastole. Thus, the roles of all individual currents (like pump and exchanger) in pacemaker activity must be considered.

The aim of this study is to precise the role of each current in the normal pacemaking of SA node pacemaker cells. To do that the nonlinear dynamics of the mathematical model of the pacemaker cell of (Djabella and Sorine, 2006) is studied by a bifurcation analysis, as well as by numerical simulations. We construct bifur- 
cation diagrams by calculating stationary points, periodic orbits, stability of the equilibrium points, Hopf bifurcation points as functions of bifurcation parameters (maximal conductances) to characterize the dynamics properties of each individual ionic components in the SA node pacemaker cell model.

A salient point of this numerical study is the illustration of the usefulness of bifurcation analysis to get insight into the mechanisms of the SA node cell pacemaker activity. The $\mathrm{Ca}^{2+}, \mathrm{K}^{+}, \mathrm{Na}^{+} / \mathrm{K}^{+}$and $\mathrm{Na}^{+} / \mathrm{Ca}^{+}$currents appear to be essential for pacemaker activity because blocking one of these currents abolishes spontaneous activity. Furthermore, in the vicinity of the control conditions, the cycle length (CL) is specially more sensitive to the background calcium current than to the others. In contrast, in our model, the $\mathrm{Na}^{+}$current would be inessential to pacemaker activity, as it only modulates action potential waveforms and does not significantly affect pacemaker frequency: reducing conductance of this current does not cause bifurcation to quiescence and its effect on the stability, dynamics and bifurcation structure of the cell model much smaller than the others.

Remark that the same kind of analysis has been conducted for more complex models of SA node pacemaker cells as in (Kurata et al., 2003), based on the model of (Kurata et al., 2002) which has 29 state variables and 18 ionic currents. The simplicity of the present model with its 8 state variables and 6 ionic currents makes possible to push further the analysis.

The paper is organized as follows: the model and the theoretical and numerical methods for bifurcation analysis are described in Section 2. Section 3 shows some simulation results. A discussion and conclusions are presented in Sections 4 and 5 respectively. The model equations are recalled in an appendix.

\section{THEORY AND METHODS}

\subsection{Mathematical Model}

We use our model of SA pacemaker cell which can reproduce the dynamic properties and bifurcation structures of real SA node cells (see fig. 2 and fig. 3) despite its reduced order. The model equations and notations are recalled in the APPENDIX.

The complete model for the normal pacemaking cell includes five membrane currents, among which $I_{K, t}$, $I_{N a, t}, I_{C a, t}$, represent the sums of all the currents through the $\mathrm{K}^{+}, \mathrm{Na}^{+}, \mathrm{Ca}^{2+}$ channels respectively. The membrane currents system also includes the exchanger and the pump currents denoted as $I_{\mathrm{NaCa}}$ and $I_{N a K}$ respectively (Endresen et al., 2000), charging the membrane capacitance $C_{m}$. The background current $I_{b, C a}$ is included in the expression of the $I_{C a, t}$ current.

As for ventricular cells, the model takes into account the main processes that regulate intracellular Calcium concentration: release and uptake by the sarcoplasmic reticulum (SR), buffering in the SR by calsequestrin (ten Tusscher et al., 2004) and in the bulk cytosol (Shannon et al., 2004) where several buffers of Calcium are in competition with the myofilament protein troponin-C. In the case of ventricular cells, this competition regulates the contractile activity. To reduce the number of variables, only two buffers have been selected (calmodulin and troponin).

\subsection{Stability and Bifurcation Analysis}

A bifurcation is a change of the number or type of attractors in a nonlinear dynamical system when some system parameters are changed. It is accompanied by a change of the stability of an attractor. At a bifurcation point, at least one eigenvalue of the Jacobian gets a zero real part. The bifurcation phenomena observed in cardiac myocytes include cessation or generation of pacemaker activity and occurrence of abnormal (irregular) dynamics (Guevara and Jongsma, 1992). We will be mainly concerned by Hopf bifurcations occuring when the Jacobian matrix has a complex conjugate pair of eigenvalues. That change of the sign of the real parts, making a limit cycle to appear or disappear. Structural stability of a dynamical system is an equivalence property of systems with sufficiently close parameters (the topological description of the dynamics do not change under small perturbations) and fails when bifurcations occur (see e.g. (Kuznetsov, 1998; Wiggins, 1990) for bifurcation theory and numerical methods for bifurcation analysis). This concept may help to understand the generic behaviour of dynamical systems in state spaces of small dimensions (this is the case for continuous time dynamical systems on compact 2-dimensional manifolds that can be precisely characterised, but already false in the 3-dimensional case). A practical way to study this stability is to analyse bifurcations, that is in general a difficult task for bifurcations depending on more than 3 parameters (the number of parameter necessary to tune, to get the bifurcation is its co-dimension).

We examine here how the stability and dynamics of our 8-state-variables cell model alter with changes in bifurcations of co-dimension 1 and construct associated bifurcation diagrams for one parameter while keeping all the other parameters at their standard values. The bifurcation parameters chosen in this study are the maximum conductances of six membrane currents.

We calculate the equilibrium points (EP) and periodic orbits in the state space and also determine the asymptotic stability of an EP by computing 8 eigenvalues of the $8 \times 8$ Jacobian matrix derived from the linearization of the nonlinear system around the EP. Then, EPs (steady state branches) and local extrema of periodic orbits (periodic branches) are plotted against the bifurcation parameter. Hopf bifurcation (HB) points where 
the stability analysis of an EP reverses were detected by the stability analysis as described above.

\subsection{Numerical Integration}

We use the software XPP (also called XPPAUT) running under X11 and Windows which is a tool for analyzing dynamical systems (Ermentrout, 2002).

Dynamic behavior of the cell model is determined by solving the simultaneous nonlinear ordinary differential equations (ODE) numerically. We use CVODE algorithm which is a solver for stiff and non-stiff ODE systems, that includes variable-order methods and variable-step methods. The maximum relative error tolerance for our integration method is set to $10^{-6}$. The action potential (AP) amplitude, the voltage difference between the maximum diastolic (MDP) and peak overshoot potential (POP), as well as the $\mathrm{CL}$, are determined for each cycle. The continuationbifurcation algorithms of XPP are used for the fixed points and periodic solutions of the ODE.

\section{RESULTS}

Figure 2 shows the spontaneous action potentials together with the underlying five membrane currents in the cell model; $I_{N a, t}, I_{C a, t}, I_{K, t}, I_{N a K}$ and $I_{N a C a}$. In Figure 3 , the modelled action potential is shown together with an experimental curve of (Baruscotti et al., 1996). The curve are identical in shape, but the modelled curve is adjusted somewhat (we multiplied the voltage amplitude by a factor 1.26 , without changing the minimum value) to obtain the same voltage amplitudes.

To identify the key currents responsible for generation and control of pacemaker activity, we explore the effects of changing the conductances of $I_{N a, t}\left(\bar{I}_{N a}\right)$, $I_{C a, t}\left(\bar{I}_{C a}\right), I_{b, C a}\left(\bar{I}_{b, C a}\right), I_{K, t}\left(\bar{I}_{K}\right), I_{N a K}\left(\bar{I}_{N a K}\right)$ and $I_{N a C a}$ $\left(\bar{I}_{\mathrm{NaCa}}\right)$ currents on EP stability and oscillation dynamics of the cell model.

Figure 1 shows the bifurcation diagrams constructed for $\bar{I}_{K}, \bar{I}_{C a}, \bar{I}_{N a K}, \bar{I}_{N a C a}$ and $\bar{I}_{b, C a}$, depicting the steady state potential $\left(V_{0}\right)$ at an EP (steady state branch) and local potential extrema of MDP and POP (periodic branches), as well as CL, as functions of bifurcation parameters. The steady state potential is negatively or positively shifted with decreasing $\bar{I}_{K}, \bar{I}_{C a}$ and $\bar{I}_{N a K}$ while it is approximately constant with decreasing $\bar{I}_{b, \mathrm{Ca}}$ or $\bar{I}_{\mathrm{NaCa}}\left(V_{0} \simeq-25 \mathrm{mV}\right)$. Decreasing $\bar{I}_{K}$ or $\bar{I}_{\mathrm{NaCa}}$ leads to stabilization of the EP: HB points are at $\bar{I}_{K}=$ $14.08 p A$ and $\bar{I}_{N a C a}=0.70 p A$ respectively, with a cessation of limit cycle oscillations. When changing $\bar{I}_{C a}$, the amplitude of a limit cycle gradually decreases and finally the oscillation disappears at the HB points. The HB points are located at $\bar{I}_{C a}=42.3 p A$ and at $\bar{I}_{C a}=$ $2.56 p A$. From the second one, an unstable branch emerging goes in the same direction as the steady state solution which loses stability (to the right). We note
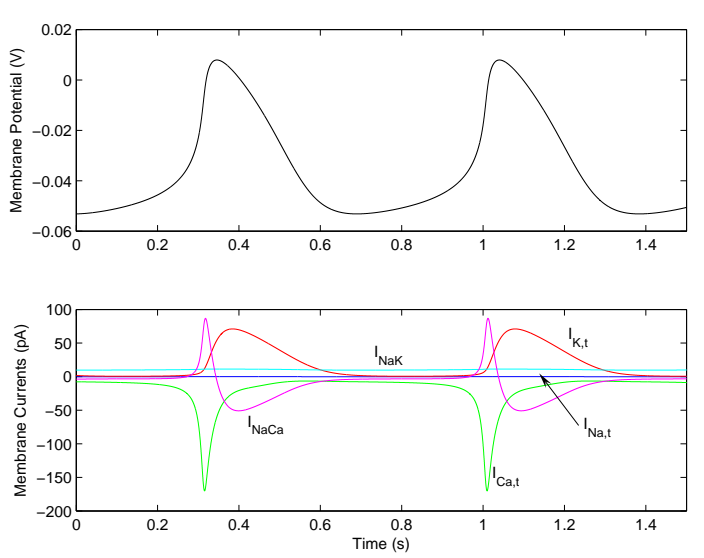

Fig. 2. Simulated dynamics of the SA node cell model. Top: model-generated AP waveform, Bottom: underlying sarcolemmal currents

that none stable solution (a periodic orbit or stationary solution) exist in a very small zone around this $\mathrm{HB}$ point (the same remark is done for the HB point at $\bar{I}_{\mathrm{NaCa}}=0.70 \mathrm{pA}$. Thus, we think that XPP could not find a sable solution, this may suggest that there is a nontrivial solution which XPP could not track.

The same behaviour as for $\bar{I}_{C a}$ is found when changing $\bar{I}_{N a K}$ : a stable limit cycle gradually decreases and finally disappears at the Hopf bifurcation points located at $\bar{I}_{N a K}=7.8 \mathrm{pA}$ and at $\bar{I}_{\mathrm{NaK}}=64.45 \mathrm{pA}$. We have find also two limit points at $\bar{I}_{N a K}=0.73 \mathrm{pA}$ and at $\bar{I}_{N a K}=0.86 p A$, the curve of EP along the middle branch is unstable. When increasing $\bar{I}_{b, C a}$, a stable limit cycle gradually decreases to desappear at the HB point $\bar{I}_{b, C a}=0.1899 p A$.

The cycle length variations are also shown as functions of the bifurcation parameters (see the right panel of the fig. 1). In the vicinity of the control conditions, the CL is especially sensitive to the change of $\bar{I}_{b, C a}$ but relatively insensitive to the others. This background calcium current has a very small conductance value,

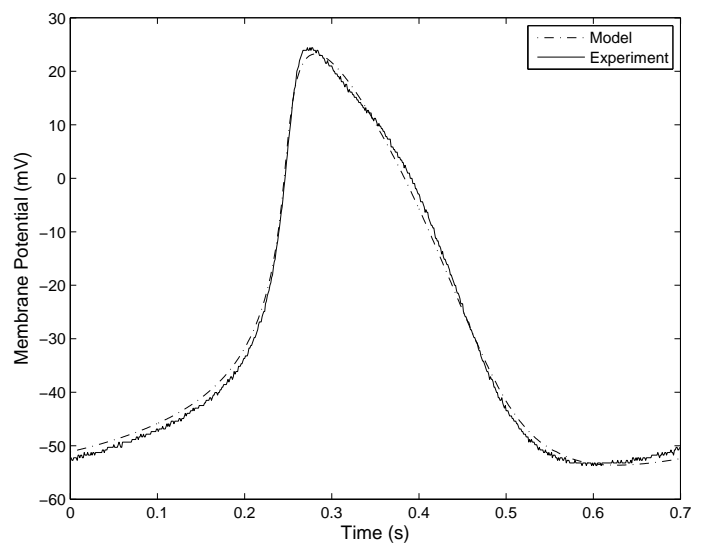

Fig. 3. Experimentally recorded and scaled (by a factor of 1.26) model-generated rabbit sinoatrial action potential waveform. 

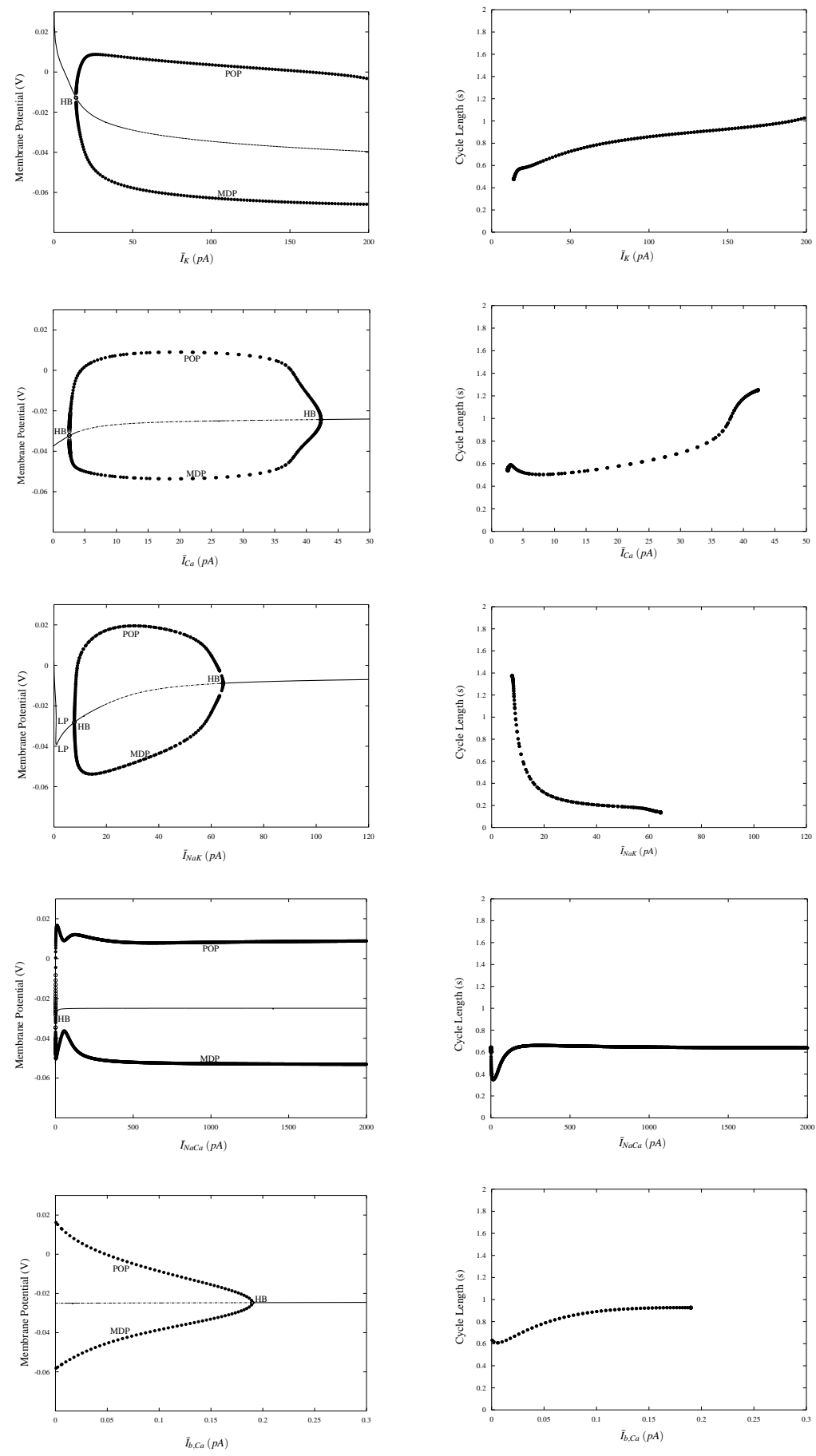

Fig. 1. Effects of changing current conductances $\bar{I}_{K}, \bar{I}_{C a}, \bar{I}_{N a K}, \bar{I}_{N a C a}$ and $\bar{I}_{b, C a}$ on EP stability and oscillation dynamics of the cell model. Bifurcation diagrams with steady state potential (stable: solide line, unstable: dashed line) and periodic potential branches (the filled circles represent MDP and POP values of stable periodic solution, and the unfilled circles represent MDP and POP values of unstable periodic branch) are constructed on the left and variations in cycle length against bifurcation parameters on the right.

and a very small change in this value causes an appreciable change in the CL.

Figure 4 shows the bifurcation diagrams constructed for $\bar{I}_{N a}$, depicting the steady state potential $\left(V_{0}\right)$ at an EP (steady state branch) and local potential extrema of MDP and POP (periodic branches), as well as CL, as functions of the bifurcation parameter. Changing $\bar{I}_{N a}$ does not stabilize the EP or abolish spontaneous activity and the CL is insensitive to its variation. 

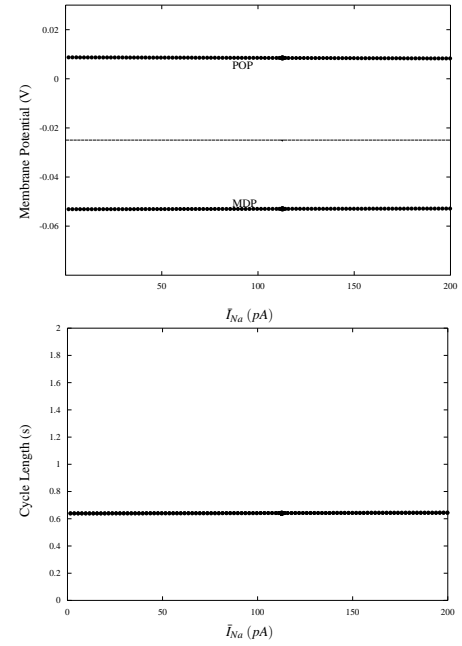

Fig. 4. Effects of changing current conductance $\bar{I}_{N a}$. Notations are similar to fig. 1.

\section{DISCUSSION}

Following the approach in (Kurata et al., 2003), we have investigated some dynamical mechanisms of the SA node pacemaker activity by a stability and bifurcation analysis of our reduced order model of pacemaker cell. The pacemaker activity corresponds here to a stable limit cycle around an unstable EP, so that the most important roles of the sarcolemmal currents in pacemaking, are their abilities to drive to the instability of an EP or contribute to maintain it, avoiding the quiescent state of the system at stable EPs (Guevara and Jongsma, 1992) as in the case of the stable resting potential of atrial or ventricular myocytes. Our results are compatible with the qualitative behaviour of the more complex model of (Kurata et al., 2003). Initiation or arrest of normal pacemaking appear as Hopf bifurcation phenomena controlled by dedicated currents.

We have considered the roles of $I_{N a, t}, I_{C a, t}, I_{b, C a}, I_{K, t}$, $I_{\mathrm{NaK}}$ and $I_{\mathrm{NaCa}}$ currents in SA node pacemaker cell by exploring how these currents affect the bifurcation structure of the cell model. Each of $I_{C a, t}, I_{b, C a}, I_{K, t}$, $I_{\mathrm{NaCa}}$ and $I_{\mathrm{NaK}}$ is able to induce to EP instability and pacemaker generation via a Hopf bifurcation. Furthermore, $I_{b, C a}$ appears to be indispensable for pacemaker activity in that it is the main responsible for cycle length sensitivity (fig. 1). EP instability and oscillation dynamics are not affected by the $I_{N a, t}$ current (Fig. 4).

\section{CONCLUSION}

In this paper, stability and bifurcation analysis is used to investigate the mechanisms of pacemaking in the SA node cell represented by a reduced order mathematical model. This nonlinear dynamical approach is known to be useful for general understanding and systematic description of the mechanisms of normal or abnormal pacemaker activity (Guevara and Jongsma, 1992). This study demonstrate that bifurcation theory also provides a convenient way of determining the roles of individual currents in pacemaker activity of SA node cells and to compare qualitative behaviours of models of various complexity (here 8 state variables against 29 in (Kurata et al., 2002)). In our reduced order model of SA node cell, pacemaker activity depends on the conductivity ranges of $I_{C a, t}, I_{b, C a}, I_{K, t}$, $I_{N a C a}$ and $I_{N a K}$ which are responsible for equilibrium points instability via a Hopf bifurcation. Furthermore, $I_{b, C a}$ allows fine tuning of the SA node rate.

This work also suggests that for some applications where reduced order models of SA node cell are required (e.g. long-term or multi-cell simulations, analysis or identification...), it is possible to check that the models used have the required qualitative behaviours (i.e. bifurcation structures) of larger SA node cell models validated against sub-cellular experiments.

\section{APPENDIX}

The model of SA pacemaker cell is the system (1) of eight ODEs (notations are defined in table 1):

$$
\left\{\begin{aligned}
\frac{d V}{d t} & =-\frac{I_{K, t}+I_{N a, t}+I_{C a, t}+I_{N a K}+I_{N a C a}}{C_{m}} \\
\frac{d K_{i}}{d t} & =\frac{2 I_{N a K}-I_{K, t}}{F V_{C}} \\
\frac{d N a_{i}}{d t} & =-\frac{I_{N a, t}+3 I_{N a K}+3 I_{N a C a}}{F V_{C}} \\
\frac{d C a_{i}}{d t} & =\frac{2 I_{N a C a}-I_{C a, t}}{2 F V_{C}}+J_{l e a k}+J_{r e l}-J_{u p} \\
- & \sum_{b \in I_{B}} B_{b} \frac{d \theta_{b}}{d t}, \quad I_{B}=\{T n, C a l\}, \\
\frac{d g_{X}}{d t} & =\frac{g_{X \infty}-g_{X}}{\tau_{g X}}, \quad X \in\{N a, K\} \\
\frac{d \theta_{b}}{d t} & =k_{b}^{o n}\left|C a_{i}\right|_{+}\left(1-\theta_{b}\right)-k_{b}^{o f f} \theta_{b}, \quad b \in I_{B}
\end{aligned}\right.
$$

The gate dynamics are defined by

$$
\begin{aligned}
g_{N a \infty} & =\frac{1}{2}\left[1-\tanh \left(\frac{V-V_{g_{N a}}}{R T / 2 F}\right)\right], \\
g_{X \infty} & =\frac{1}{2}\left[1+\tanh \left(\frac{V-V_{g_{X}}}{R T / 2 F}\right)\right], \quad X \in\{K, d, m\} \\
\frac{1}{\tau_{g_{X}}} & =\frac{1}{\tau_{X}} \cosh \left(\frac{V-V_{g_{X}}}{R T / 2 F}\right), \quad X \in\{N a, K\}
\end{aligned}
$$

where $X=d, m$ represent fast $\mathrm{Ca}, \mathrm{Na}$ activation gating, denoted as usual $d_{\infty}=g_{d \infty}, m_{\infty}=g_{m \infty}$. Setting

$$
V_{X}=\frac{R T}{z_{X} F} \log \left|\frac{X_{e}}{X_{i}}\right|, X \in\{C a, N a, K\}
$$

the currents through the membrane are then: 
Table 1. Notations used in the text

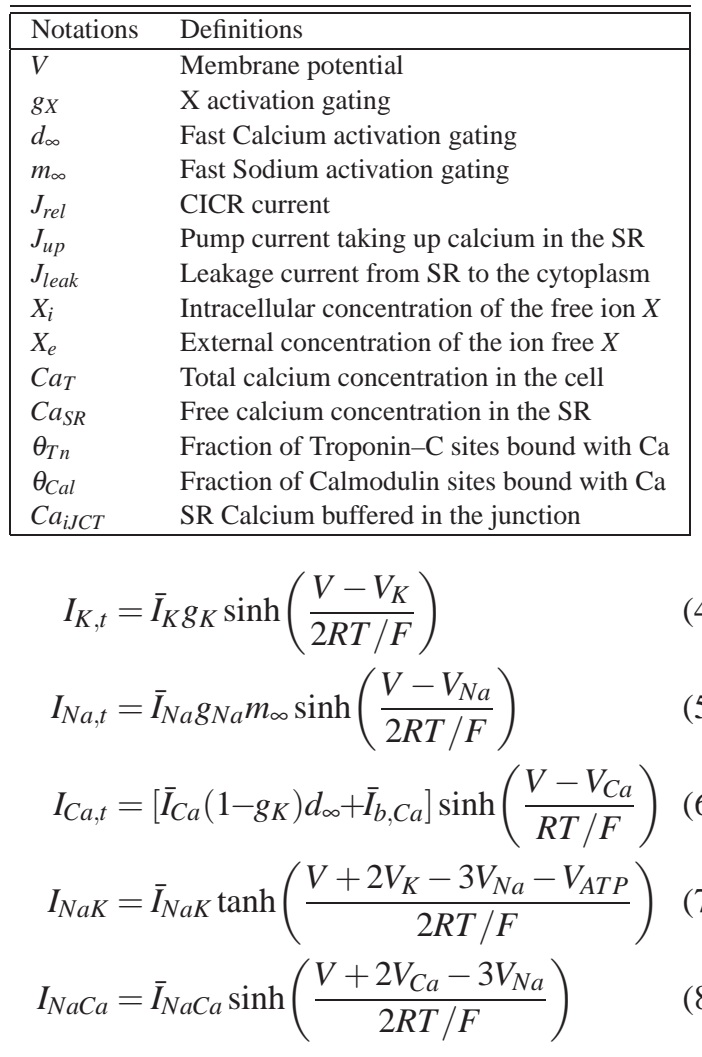

Finally, the calcium-induced calcium release (CICR) mechanism is described using

$$
\begin{aligned}
& J_{\text {rel }}= K_{\text {rel }} d_{\infty}\left(C a_{S R}-C a_{i J C T}\right) \\
& J_{\text {leak }}=K_{\text {leak }}\left(C a_{S R}-C a_{i J C T}\right) \\
& C a_{i J C T}= \frac{B_{J C T}\left|C a_{i}\right|_{+}}{\left|C a_{i}\right|_{+}+K_{J C T}} \\
& C a_{S R}+ \frac{B_{S R}\left|C a_{S R}\right|_{+}}{\left|C a_{S R}\right|_{+}+K_{S R}}= \\
& \frac{V_{C}}{V_{S R}}\left(C a_{T}-C a_{i}-\sum_{b \in I_{B}} B_{b} \theta_{b}-C a_{i J C T}\right) \\
& C a_{T}=C a_{e}+\frac{C_{m}}{2 F V_{C}} V+\frac{1}{2}\left(N a_{e}-N a_{i}+K_{e}-K_{i}\right)
\end{aligned}
$$

\section{REFERENCES}

Baruscotti, M., D. DiFrancesco and R. B. Robinson (1996). A ttx-sensitive inward sodium current contributes to spontaneous activity in newborn rabbit sino-atrial node cells. $J$ Physiol 492, 2130.
Boyett, M. R., H. Zhang, A. Garny and A. V. Holden (2001). Control of the pacemaker activity of the sinoatrial node by intracellular $\mathrm{ca}^{2+}$. experiments and modelling. Phil. Trans. The Royal Society 359, 1091-1110.

DiFrancesco, D. (1993). Pacemaker mechanisms in cardiac tissue. Annual Review of Physiol. 55, 455-472.

Djabella, K. and M. Sorine (2006). A differential model of controlled cardiac pacemaker cell. In: IFAC Modelling and Control in Biomedical Systems. Reims, France. pp. 459-464.

Endresen, L. P., K. Hall, J. S. Hoye and J. Myrheim (2000). A theory for the membrane potential of living cells. Eur Biophys J 29, 90-103.

Ermentrout, B. (2002). Simulating, analyzing, and animating dynamical system. Siam. Philadelphia.

Guevara, M. R. and H. J. Jongsma (1992). Three ways of abolishing automaticity in sinoatrial node: ionic modeling and nonlinear dynamics. Am $J$ Physiol Heart Circ Physiol 262, H1268-1286.

Guo, J., K. Ono and A. Noma (1995). A sustained inward current activated at the diastolic potential range in rabbit sinoatrial node cells. Journal of Physiol. 483, 1-13.

Kodama, I., H. Honjo and M. R. Boyett (2002). Are we lost in the labyrinth of the sinoatrial node pacemaker mechanism?. J. Cardiovascular Electrophysiology 13, 1303-1305.

Kurata, Y., I. Hisatome, S. Imanishi and T. Shibamoto (2002). Dynamical description of sinoatrial node pacemaking: improved mathematical model for primary pacemaker cell. Am J Physiol Heart Circ Physiol 283(5), H2074-2101.

Kurata, Y., I. Hisatome, S. Imanishi and T. Shibamoto (2003). Roles of L-type Ca2+ and delayedrectifier $\mathrm{K}+$ currents in sinoatrial node pacemaking: insights from stability and bifurcation analyses of a mathematical model. Am J Physiol Heart Circ Physiol 285(6), H2804-2819.

Kuznetsov, Y. A. (1998). Elements of applied bifurcation theory (2nd ed.). Springer. New York.

Shannon, T. R., F. Wang, J. Puglisi, C. Weber and D. M. Bers (2004). A mathematical treatment of integrated ca dynamics within the ventricular myocyte. Biophys $J$ 87, 3351-3371.

ten Tusscher, K. H. W. J., D. Noble, P. J. Noble and A. V. Panfilov (2004). A model for human ventricular tissue. Am J Physiol Heart Circ Physiol 286, 1573-1589.

Wiggins, S. (1990). Introduction to applied nonlinear dynamical systems and chaos. Springer. New York. 